\title{
Rank 2 preservers on symmetric matrices with zero trace
}

\author{
Wai Keong $\mathrm{Kok}^{1 *}$ \\ ${ }^{1}$ Department of Mathematical and Data Science, Faculty of Computing and Information Technology, \\ Tunku Abdul Rahman University College 53300 Kuala Lumpur, Malaysia
}

\begin{abstract}
Let $F$ be a field, $V_{1}$ and $V_{2}$ be vector spaces of matrices over $F$ and let $\rho$ be the rank function. If $T: V_{1} \rightarrow V_{2}$ is a linear map, and $k$ a fixed positive integer, we say that $T$ is a rank $k$ preserver if for any matrix $A \in V_{1}$, $\rho(A)=k$ implies $\rho(T(A))=k$. In this paper, we characterize those rank 2 preservers on symmetric matrices with zero trace under certain conditions.
\end{abstract}

\section{Introduction}

Let $F^{n \times n}$ be the algebra of all $n \times n$ matrices over a field $F$. Let $s l_{n}(F)$ denote the subspace of $F^{n \times n}$ consisting of all matrices with zero trace. In [1], Botta, Pierce and Watkins obtained a useful result concerning the structure of nonsingular linear mapping on $s l_{n}(F)$ that preserve nilpotent matrices where $F$ is infinite. In [2], Li and Pierce characterized linear mappings on $s l_{n}(F)$ that preserve nonzero nilpotent matrices with rank at most $k$ where $k$ is a fixed positive integer less than $n$ and $F$ is algebraically closed of characteristic zero. Then, Watkins characterized linear mappings from $s l_{n}(F)$ to $F^{n \times n}$ that preserve rank one matrices where $F$ is an algebraically closed field of characteristic not equal to 2 . He applied this result to determine the structure of bilinear mappings on $F^{n \times n}$ that have certain rank-preserving properties in [3] and [4] respectively.

Let $S_{n}(F)$ be the vector space of all $n \times n$ symmetric matrices over $F$ and $Z_{0}\left(S_{n}(F)\right)$ be its subspace consisting of all symmetric matrices with zero trace. Let $n \geq 4$ and $F$ be a field of characteristic greater than 3. Motivated by work of Lim [5] in the characterization of linear rank one preservers on matrices with zero trace, we characterize those rank 2 preservers on symmetric matrices with zero trace under certain conditions in this paper and will discuss some consequences of this characterization in our next paper.

*Corresponding author: kokwk@tarc.edu.my 


\section{Some definitions and preliminary results}

Let $U$ be a vector space over $F$. We use tensor language in our investigation. This provides us with a larger context. We denote by $U^{(2)}$ the second symmetric product space over $U$ and denoted by $x \cdot y, x, y \in U$, the decomposable elements of $U^{(2)}$. For each $u$ in $U$, let $u^{2}$ denote $u \cdot u$.

A scalar product on $U$ is a function which assigns a scalar $(x, y) \in F$ to each ordered pair of vectors $x, y \in U$ such that for any $x, y, z \in U$ and any $c \in F$

(i) $\quad(x+y, z)=(x, z)+(y, z)$

(ii) $\quad(c x, y)=c(x, y)$

(iii) $(x, y)=(y, x)$

We say $x$ is orthogonal to $y$ or $x$ and $y$ are orthogonal if $(x, y)=0$. Let $S$ be a set of vectors in $U$. Then $S$ is called an orthogonal set if $(x, y)=0$ for all $x, y \in S, x \neq y$. If in addition, $(x, x)=1$ for every $x \in S$, then $S$ is called an orthonormal set.

Now we let $U$ be equipped with a scalar product (,): $U \times U \rightarrow F$ and $U$ has an orthonormal basis $\mathcal{E}$. Let $Z_{0}\left(U^{(2)}\right)$ be the subset of $U^{(2)}$ that consists of all vectors of the form

$$
\sum_{1 \leq i \leq j \leq n} a_{i j} u_{i} \cdot u_{j}
$$

where $\left\{u_{1}, \ldots, u_{n}\right\}$ is an arbitrary finite subset of $\mathcal{E}$ and $a_{i j}(1 \leq i \leq j \leq n)$ are arbitrary scalars in $F$ such that $\sum_{i=1}^{n} a_{i i}=0$. Clearly $Z_{0}\left(U^{(2)}\right)$ is a subspace of $U^{(2)}$ and we call $Z_{0}\left(U^{(2)}\right)$ the space of traceless $2^{\text {nd }}$ order symmetric tensors over $U$.

Proposition 2.1 If $\left\{e_{i}: i \in A\right\}$ where $A \supseteq\{1,2\}$ is an orthonormal basis for $U$, then $B=\left\{e_{i} \cdot e_{j}, e_{1}^{2}-e_{k}^{2}: i \neq j, k \neq 1\right.$ and $\left.1, i, j, k \in A\right\}$ is a basis for $Z_{0}\left(U^{(2)}\right)$.

\section{Proof.}

Clearly $B$ is a linearly independent set. Hence it is sufficient to show that $B$ spans $Z_{0}\left(U^{(2)}\right)$.

Let $x \in Z_{0}\left(U^{(2)}\right)$. Then $x=\sum_{1 \leq i \leq j \leq n} a_{i j} u_{i} \cdot u_{j}$ and $\sum_{i=1}^{n} a_{i i}=0$ where $\left\{u_{1}, \ldots, u_{n}\right\}$ is a finite subset of $\left\{e_{i}: i \in A\right\}$ and $a_{i j}(1 \leq i \leq j \leq n)$ are scalars in $F$. It follows that

$$
x=\sum_{1 \leq i \leq j \leq n} a_{i j} u_{i} \cdot u_{j}-\sum_{k=1}^{n} a_{k k}\left(e_{1}^{2}-u_{k}^{2}\right) .
$$

Therefore, $B$ spans $Z_{0}\left(U^{(2)}\right)$.

Let $Z_{0}\left(S_{n}(F)\right)$ denote the subspace of $S_{n}(F)$ such that for any $A \in Z_{0}\left(S_{n}(F)\right)$, $\operatorname{tr}(A)=0$. If $U$ is a finite dimensional vector space with an orthonormal basis $\left\{e_{i}: i=1, \ldots, n\right\}$, then $Z_{0}\left(U^{(2)}\right)$ is isomorphic in a natural way to $Z_{0}\left(S_{n}(F)\right)$ by the restricted 
isomorphism $\left.\varphi\right|_{Z_{0}\left(U^{(2)}\right)}$ where $\varphi$ is the isomorphism from $U^{(2)}$ to $S_{n}(F)$ defined by $\varphi\left(e_{i} \cdot e_{j}\right)=E_{i j}+E_{j i}, 1 \leq i \leq j \leq n$.

Remark. If $U$ is a Euclidean space, then there does not exist any rank 1 vector in $Z_{0}\left(U^{(2)}\right)$.

Let $J_{k}$ denote the set of vectors in $U^{(2)}$ of the form $\sum_{i=1}^{k} \lambda_{i} x_{i}^{2}$, where $x_{1}, \ldots, x_{k}$ are linearly independent vectors and $\lambda_{1}, \ldots, \lambda_{k} \in F \backslash\{0\}$. For each vector $u \in U$, let $u \cdot U=\{u \cdot v: v \in U\}$.

Lemma 2.2 Let $M$ be a subspace of $U^{(2)}$ such that $M \subseteq\{0\} \cup J_{1} \cup J_{2}$. Then either

(i) $M \subseteq W^{(2)}$ for some subspace $W$ of $U$ that is 2 dimension or

(ii) $M \subseteq u \cdot U$ for some $u \in U \backslash\{0\}$.

\section{Proof.}

If $M \cap J_{2}=\varnothing$, then clearly $M=\left\langle x^{2}\right\rangle$ for some $x$ in $U$. Let $A_{1}=a u_{1}^{2}+u_{2}^{2} \in J_{2} \cap M$. Assume that $M \not \subset V^{(2)}$ where $V=\left\langle u_{1}, u_{2}\right\rangle$. Then it is clear that there exists $A_{2}=b u_{3}^{2}+v^{2} \in J_{2} \cap M$ where $u_{1}, u_{2}, u_{3}$ are linearly independent. Clearly $v \in\left\langle u_{1}, u_{2}, u_{3}\right\rangle$, otherwise $A_{1}+A_{2} \in J_{4}$, a contradiction. Let $v=c u_{1}+d u_{2}+e u_{3}$ where $c, d, e \in F$.

Since for any $\lambda \in F$,

$$
\begin{aligned}
\lambda A_{1}+A_{2}= & \left(\lambda a+c^{2}\right) u_{1}^{2}+\left(\lambda+d^{2}\right) u_{2}^{2}+\left(b+e^{2}\right) u_{3}^{2} \\
& +2 c d u_{1} \cdot u_{2}+2 c e u_{1} \cdot u_{3}+2 d e u_{2} \cdot u_{3} \in J_{1} \cup J_{2},
\end{aligned}
$$

it follows that

$$
\begin{aligned}
& \left|\begin{array}{ccc}
\lambda a+c^{2} & c d & c e \\
c d & \lambda+d^{2} & d e \\
c e & d e & b+e^{2}
\end{array}\right| \\
& =a\left(b+e^{2}\right) \lambda^{2}+b\left(a d^{2}+c^{2}\right) \lambda \\
& =0
\end{aligned}
$$

for any $\lambda \in F$. Since $|F| \geq 3$, we have

$$
b+e^{2}=0
$$

and

From (1) we have

$$
a d^{2}+c^{2}=0 .
$$

$$
A_{2}=\left(c u_{1}+d u_{2}\right) \cdot\left(c u_{1}+d u_{2}+2 e u_{3}\right) .
$$

From (2) we have $a d c^{-1}+d^{-1} c=0$ and hence

$$
A_{1}=\left(c u_{1}+d u_{2}\right) \cdot\left(a c^{-1} u_{1}+d^{-1} u_{2}\right) .
$$

From (3) and (4) we have $A_{1}=u \cdot y_{1}, A_{2}=u \cdot y_{2}$ where $u, y_{1}, y_{2}$ are linearly independent.

Suppose now $A=x^{2} \in J_{1} \cap M$. Then $A+A_{1}, A+A_{2} \in J_{1} \cap J_{2}$ imply that $x \in\left\langle u, y_{1}\right\rangle \cap\left\langle u, y_{2}\right\rangle=\langle u\rangle$ and hence $A \in u \cdot U$.

Suppose that $B=\lambda_{1} v_{1}^{2}+\lambda_{2} v_{2}^{2} \in J_{2} \cap M$. For each $\lambda \in F$, let $C_{\lambda}=u \cdot z_{\lambda}$, where $z_{\lambda}=y_{1}+\lambda y_{2}$. Then $C_{\lambda} \in M$. Clearly there exists a subset $D$ of $F$ with 2 elements such 
that $\left\langle v_{1}, v_{2}\right\rangle \neq\left\langle u, z_{\lambda}\right\rangle$ for all $\lambda \in D$. Hence $\operatorname{dim}\left\langle v_{1}, v_{2}, u, z_{\lambda}\right\rangle \geq 3$ for all $\lambda \in D$. By our previous argument, for any $\lambda \in D$, there exists $w_{\lambda} \in U$ such that

$$
B, C_{\lambda} \in w_{\lambda} \cdot U \text {. }
$$

Suppose $B \neq u \cdot U$. Then

$$
B=\alpha z_{i} \cdot z_{j}
$$

for some $\alpha \in F \backslash\{0\}$ where $i$ and $j$ are distinct element in $D$. Let

$$
H=u \cdot z_{k}
$$

where $k \in F \backslash\{i, j\}$. Since $\operatorname{dim}\left\langle u, z_{i}, z_{j}, z_{k}\right\rangle=3$,

$$
B, H \in v \cdot U
$$

for some $v \in U$ by previous argument. This yields a contradiction since $B=\alpha z_{i} \cdot z_{j}$ and $H=u \cdot z_{k}$ do not have a common factor. Therefore $B=u \cdot U$.

\section{Rank 2 preservers}

Let $U$ and $W$ be vector spaces over $F$. We always assume that $U$ has an orthonormal basis, $\left\{e_{i}: i \in \boldsymbol{A}\right\}$, with respect to a scalar product $():, U \times U \rightarrow F$, where $\boldsymbol{A} \supseteq\{1,2, \ldots, n\}$ if $\boldsymbol{A}$ has at least $n$ elements. For each vector $u \in U$, let $\langle u\rangle^{\perp}=\{v \in U:(v, u)=0\}$.

Lemma 3.1 Let $T: Z_{0}\left(U^{(2)}\right) \rightarrow W^{(2)}$ be a rank 2 preserver. If $V$ is a subspace of $U$ such that $V \subseteq\langle u\rangle^{\perp}$ for some $u \in U \backslash V$, then $\operatorname{dim} T(u \cdot V)=\operatorname{dim} u \cdot V$. Moreover, if $\operatorname{dim} V \geq 3$, then $T(u \cdot V) \subseteq w \cdot W$ for some $w \in W \backslash\{0\}$.

\section{Proof.}

Suppose $T\left(u \cdot v_{1}\right)=T\left(u \cdot v_{2}\right)$ for some $v_{1}, v_{2} \in V$. Then $T\left(u \cdot\left(v_{1}-v_{2}\right)\right)=0$ and this implies that $v_{1}=v_{2}$, since $T$ is a rank 2 preserver. Hence $\operatorname{dim} T(u \cdot V)=\operatorname{dim} u \cdot V$. If $\operatorname{dim} V \geq 3$, then $\operatorname{dim}(T(u \cdot V)) \geq 3$. Since $T(u \cdot V)$ is a subspace of $W^{(2)}$ contained in $J_{2} \cup\{0\}$, it follows by Lemma 2.2 that $T(u \cdot V) \subseteq w \cdot W$ for some $w \in W \backslash\{0\}$.

Theorem 3.2 Let $T$ be a rank 2 preserver from $Z_{0}\left(U^{(2)}\right)$ to $W^{(2)}$. If $\operatorname{dim} U \geq 4$, then one of the following holds:

(i) $T=\left.\lambda P_{2}(f)\right|_{Z_{0}\left(U^{(2)}\right)}$ for some $\lambda \in F \backslash\{0\}$ and some one-to-one linear mapping $f: U \rightarrow W$ where $P_{2}(f)$ is a second induced power of $f$ such that $P_{2}(f)(x \cdot y)=f(x) \cdot f(y)$;

(ii) $\operatorname{Im} T \subseteq w \cdot W$ for some $w \in W \backslash\{0\}$. 


\section{Proof.}

Note that $\left\langle e_{i}\right\rangle^{\perp}$ is a subspace of $U, \operatorname{dim}\left\langle e_{i}\right\rangle^{\perp} \geq 3$ and $e_{i} \notin\left\langle e_{i}\right\rangle^{\perp}$. In view of Lemma 3.1, $T\left(e_{i} \cdot\left\langle e_{i}\right\rangle^{\perp}\right) \subseteq w_{i} \cdot W$ for some $w_{i} \in W \backslash\{0\}, i \in A$. Now we have either $\left\{w_{i}: i \in A\right\}$ is a pairwise linearly independent set or $\left\langle w_{i}\right\rangle=\left\langle w_{j}\right\rangle$ for some distinct $i, j$. We will consider these two cases separately.

Case 1: $\left\{w_{i}: i \in A\right\}$ is a pairwise linearly independent set.

Since $T\left(e_{1} \cdot e_{2}\right) \in w_{1} \cdot W, T\left(e_{2} \cdot e_{1}\right) \in w_{2} \cdot W$ and $w_{1}, w_{2}$ are linearly independent, we have $T\left(e_{1} \cdot e_{2}\right)=\alpha_{12} w_{1} \cdot w_{2}$ for some $\alpha_{12} \in F \backslash\{0\}$. Likewise,

$$
T\left(e_{i} \cdot e_{j}\right)=\alpha_{i j} w_{i} \cdot w_{j}
$$

where $\alpha_{i j} \in F \backslash\{0\}$ for all distinct $i, j$. Clearly

$$
\alpha_{i j}=\alpha_{j i} .
$$

Now we claim that $\left\{w_{i}: i \in A\right\}$ is a linearly independent set. Suppose the contrary. Let $w_{1}=\sum_{i \in A \backslash\{1\}} a_{i} w_{i}$ for some $a_{i} \in F$. Then from (5), we have

$$
\begin{aligned}
T\left(e_{2} \cdot\left(\frac{1}{\alpha_{12}} e_{1}-\sum_{i \in A \backslash\{1,2\}} \frac{a_{i}}{\alpha_{i 2}} e_{i}\right)\right) & =w_{2} \cdot\left(w_{1}-\sum_{i \in A \backslash\{1,2\}} a_{i} w_{i}\right) \\
& =a_{2} w_{2}^{2}
\end{aligned}
$$

is of rank $\leq 1$, a contradiction. So, $\left\{w_{i}: i \in A\right\}$ is a linearly independent set. Let

$$
M=\left\langle\left(e_{1}+e_{2}\right) \cdot\left(e_{1}-e_{2}\right),\left(e_{1}+e_{2}\right) \cdot e_{3},\left(e_{1}+e_{2}\right) \cdot e_{4}\right\rangle .
$$

Since $\left\langle e_{1}-e_{2}, e_{3}, e_{4}\right\rangle$ is a 3-dimensional subspace of $\left\langle e_{1}+e_{2}\right\rangle^{\perp}$ and $e_{1}+e_{2} \notin\left\langle e_{1}-e_{2}, e_{3}, e_{4}\right\rangle$, it follows by Lemma 3.1 that $\operatorname{dim} T(M)=3$ and $T(M) \subseteq u \cdot W$ for some $u \in W \backslash\{0\}$.

Let

$$
\begin{array}{r}
T\left(\left(e_{1}+e_{2}\right) \cdot\left(e_{1}-e_{2}\right)\right)=u \cdot u_{1}, \\
T\left(\left(e_{1}+e_{2}\right) \cdot e_{3}\right)=u \cdot u_{2}, \\
T\left(\left(e_{1}+e_{2}\right) \cdot e_{4}\right)=u \cdot u_{3},
\end{array}
$$

where $\operatorname{dim}\left\langle u_{1}, u_{2}, u_{3}\right\rangle=3$. In view of (5), we have

$$
\begin{aligned}
T\left(\left(e_{1}+e_{2}\right) \cdot e_{3}\right) & =T\left(e_{1} \cdot e_{3}\right)+T\left(e_{2} \cdot e_{3}\right) \\
& =\left(\alpha_{13} w_{1}+\alpha_{23} w_{2}\right) \cdot w_{3}, \\
T\left(\left(e_{1}+e_{2}\right) \cdot e_{4}\right) & =T\left(e_{1} \cdot e_{4}\right)+T\left(e_{2} \cdot e_{4}\right) \\
& =\left(\alpha_{14} w_{1}+\alpha_{24} w_{2}\right) \cdot w_{4} .
\end{aligned}
$$

Hence

$$
\begin{aligned}
& u \cdot u_{2}=\left(\alpha_{13} w_{1}+\alpha_{23} w_{2}\right) \cdot w_{3}, \\
& u \cdot u_{3}=\left(\alpha_{14} w_{1}+\alpha_{24} w_{2}\right) \cdot w_{4} .
\end{aligned}
$$

Since $\operatorname{dim}\left\langle w_{1}, w_{2}, w_{3}, w_{4}\right\rangle=4$, we have $\langle u\rangle=\left\langle\alpha_{13} w_{1}+\alpha_{23} w_{3}\right\rangle=\left\langle\alpha_{14} w_{1}+\alpha_{24} w_{2}\right\rangle$. Hence $\frac{\alpha_{23}}{\alpha_{13}}=\frac{\alpha_{24}}{\alpha_{14}}$. Likewise 


$$
\frac{\alpha_{i k}}{\alpha_{j k}}=\frac{\alpha_{i l}}{\alpha_{j l}}
$$

for all distinct $i, j, k, l$. Now let

$$
N=\left\langle\left(e_{1}-e_{2}\right) \cdot\left(e_{1}+e_{2}\right),\left(e_{1}-e_{2}\right) \cdot e_{3},\left(e_{1}-e_{2}\right) \cdot e_{4}\right\rangle .
$$

Since $\left\langle e_{1}+e_{2}, e_{3}, e_{4}\right\rangle$ is a 3 -dimensional subspace of $\left\langle e_{1}-e_{2}\right\rangle^{\perp}$ and $e_{1}-e_{2} \notin\left\langle e_{1}+e_{2}, e_{3}, e_{4}\right\rangle$, it follows by Lemma 3.1 that $\operatorname{dim} T(N)=3$ and $T(N) \subseteq v \cdot W, v \in W \backslash\{0\}$. Let

$$
\begin{aligned}
T\left(\left(e_{1}-e_{2}\right) \cdot\left(e_{1}+e_{2}\right)\right) & =v \cdot v_{1}, \\
T\left(\left(e_{1}-e_{2}\right) \cdot e_{3}\right) & =v \cdot v_{2}, \\
T\left(\left(e_{1}-e_{2}\right) \cdot e_{4}\right) & =v \cdot v_{3} .
\end{aligned}
$$

where $\operatorname{dim}\left\langle v_{1}, v_{2}, v_{3}\right\rangle=3$. On the other hand, In view of (5)

$$
\begin{aligned}
T\left(\left(e_{1}-e_{2}\right) \cdot e_{3}\right) & =T\left(e_{1} \cdot e_{3}\right)-T\left(e_{2} \cdot e_{3}\right) \\
& =\left(\alpha_{13} w_{1}-\alpha_{23} w_{2}\right) \cdot w_{3}, \\
T\left(\left(e_{1}-e_{2}\right) \cdot e_{4}\right) & =T\left(e_{1} \cdot e_{4}\right)-T\left(e_{2} \cdot e_{4}\right) \\
& =\left(\alpha_{14} w_{1}-\alpha_{24} w_{2}\right) \cdot w_{4} .
\end{aligned}
$$

Hence

$$
\begin{aligned}
& v \cdot v_{2}=\left(\alpha_{13} w_{1}-\alpha_{23} w_{2}\right) \cdot w_{3}, \\
& v \cdot v_{3}=\left(\alpha_{14} w_{1}-\alpha_{24} w_{2}\right) \cdot w_{4} .
\end{aligned}
$$

Since $\operatorname{dim}\left\langle w_{1}, w_{2}, w_{3}, w_{4}\right\rangle=4$, we have

$$
\langle v\rangle=\left\langle\alpha_{13} w_{1}-\alpha_{23} w_{3}\right\rangle=\left\langle\alpha_{14} w_{1}-\alpha_{14} w_{2}\right\rangle .
$$

Now we have

$$
T\left(\left(e_{1}+e_{2}\right) \cdot\left(e_{1}-e_{2}\right)\right) \in\left(\alpha_{13} w_{1}+\alpha_{23} w_{2}\right) \cdot W
$$

and

$$
T\left(\left(e_{1}-e_{2}\right) \cdot\left(e_{1}+e_{2}\right)\right) \in\left\langle\alpha_{13} w_{1}-\alpha_{23} w_{2}\right\rangle \cdot W .
$$

Since $\alpha_{13} w_{1}+\alpha_{23} w_{2}$ and $\alpha_{13} w_{1}-\alpha_{23} w_{2}$ are linearly independent, we have

$$
\begin{aligned}
T\left(e_{1}^{2}-e_{2}^{2}\right) & =T\left(\left(e_{1}+e_{2}\right) \cdot\left(e_{1}-e_{2}\right)\right) \\
& =\lambda_{12}^{(3)}\left(\alpha_{13} w_{1}+\alpha_{23} w_{2}\right) \cdot\left(\alpha_{13} w_{1}-\alpha_{23} w_{2}\right) \\
& =\lambda_{12}^{(3)}\left(\alpha_{13}^{2} w_{1}^{2}-\alpha_{23}^{2} w_{2}^{2}\right)
\end{aligned}
$$

where $\lambda_{12}^{(3)} \in F \backslash\{0\}$. Likewise

$$
T\left(e_{i}^{2}-e_{j}^{2}\right)=\lambda_{i j}^{(k)}\left(\alpha_{i k}^{2} w_{i}^{2}-\alpha_{j k}^{2} w_{j}^{2}\right)
$$

where $\lambda_{i j}^{(k)} \in F \backslash\{0\}$ for all distinct $i, j, k$. As a consequence

$$
\begin{gathered}
\lambda_{i j}^{(k)} \alpha_{i k}^{2}=\lambda_{i j}^{(l)} \alpha_{i l}^{2} \\
\lambda_{i j}^{(k)}=\lambda_{j i}^{(k)}
\end{gathered}
$$

for all distinct $i, j, k, l$. Now let

$$
\begin{aligned}
& u_{1}=e_{1}+e_{2}+e_{3}, \\
& u_{2}=e_{1}-e_{2}, \\
& u_{3}=e_{1}-e_{3}, \\
& u_{4}=e_{4} .
\end{aligned}
$$


Let $H=\left\langle u_{1} \cdot u_{2}, u_{1} \cdot u_{3}, u_{1} \cdot u_{4}\right\rangle$. Since $\left\langle u_{2}, u_{3}, u_{4}\right\rangle$ is a 3 -dimensional subspace of $\left\langle u_{1}\right\rangle^{\perp}$ and $u_{1} \notin\left\langle u_{2}, u_{3}, u_{4}\right\rangle$, it follows by Lemma 3.1 that $\operatorname{dim} T(H)=3$ and $T(H) \subseteq v \cdot W$ for some $v \in W \backslash\{0\}$. In view of (5), (7) and (8), we have

$$
\begin{aligned}
T\left(u_{1} \cdot u_{2}\right) & =T\left(e_{1}^{2}-e_{2}^{2}+e_{1} \cdot e_{3}-e_{2} \cdot e_{3}\right) \\
& =\lambda_{12}^{(3)}\left(\alpha_{13}^{2} w_{1}^{2}-\alpha_{23}^{2} w_{2}^{2}\right)+\alpha_{13} w_{1} \cdot w_{3}-\alpha_{23} w_{2} \cdot w_{3} \\
& =\left(\alpha_{13} w_{1}-\alpha_{23} w_{2}\right) \cdot\left(\lambda_{12}^{(3)} \alpha_{13} w_{1}+\lambda_{12}^{(3)} \alpha_{23} w_{2}+w_{3}\right) \\
T\left(u_{1} \cdot u_{3}\right) & =T\left(e_{1}^{2}-e_{3}^{2}+e_{1} \cdot e_{2}-e_{3} \cdot e_{2}\right) \\
& =\lambda_{13}^{(3)}\left(\alpha_{12}^{2} w_{1}^{2}-\alpha_{32}^{2} w_{3}^{2}\right)+\alpha_{12} w_{1} \cdot w_{2}-\alpha_{23} w_{3} \cdot w_{2} \\
& =\left(\alpha_{12} w_{1}-\alpha_{32} w_{3}\right) \cdot\left(\lambda_{13}^{(2)} \alpha_{12} w_{1}+\lambda_{13}^{(2)} \alpha_{32} w_{3}+w_{2}\right)
\end{aligned}
$$

Since $\left(\alpha_{13} w_{1}-\alpha_{23} w_{2}\right),\left(\alpha_{12} w_{1}-\alpha_{32} w_{3}\right)$ and $\left(\lambda_{12}^{(3)} \alpha_{13} w_{1}+\lambda_{12}^{(3)} \alpha_{23} w_{2}+w_{3}\right)$ are pairwise linearly independent and $T\left(u_{1} \cdot u_{2}\right), T\left(u_{1} \cdot u_{3}\right)$ have a common factor, we have

$$
\left\langle\lambda_{12}^{(3)} \alpha_{13} w_{1}+\lambda_{12}^{(3)} \alpha_{23} w_{2}+w_{3}\right\rangle=\left\langle\lambda_{13}^{(2)} \alpha_{12} w_{1}+w_{2}+\lambda_{13}^{(2)} \alpha_{32} w_{3}\right\rangle \text {. }
$$

Hence

$$
\frac{\lambda_{12}^{(3)} \alpha_{13}}{\lambda_{12}^{(2)} \alpha_{12}}=\frac{\lambda_{12}^{(3)} \alpha_{23}}{1}=\frac{1}{\lambda_{13}^{(2)} \alpha_{32}} .
$$

In view of (6), we have

$$
\begin{aligned}
& \frac{1}{\lambda_{12}^{(3)}}=\lambda_{13}^{(2)} \alpha_{23} \alpha_{32}=\lambda_{13}^{(2)} \alpha_{32}^{2}, \\
& \lambda_{13}^{(2)}=\frac{\alpha_{13}}{\alpha_{12} \alpha_{23}} .
\end{aligned}
$$

Likewise,

$$
\begin{array}{r}
\frac{1}{\lambda_{i j}^{(k)}}=\lambda_{i k}^{(j)} \alpha_{k j}^{2} \\
\lambda_{i j}^{(k)}=\frac{\alpha_{i j}}{\alpha_{i k} \alpha_{k j}}
\end{array}
$$

for all distinct $i, j, k$. In view of (12) and (7), we have

$$
\lambda_{i j}^{(k)}=\frac{1}{\alpha_{i k}}\left(\frac{\alpha_{i j}}{\alpha_{k j}}\right)=\frac{1}{\alpha_{i k}}\left(\frac{\alpha_{i l}}{\alpha_{k l}}\right)=\lambda_{i l}^{(k)}
$$

for all distinct $i, j, k, l$. In view of (10) and (13), we find that

$$
\lambda_{i j}^{(k)} \text { have a common value } \lambda^{(k)}
$$

for all $i, j \notin\{k\}$. Now we define a linear mapping $f: U \rightarrow W$ by

$$
f\left(e_{i}\right)=\left\{\begin{array}{l}
\frac{1}{\lambda} w_{3}, i=3 \\
\alpha_{i 3} w_{i}, i \neq 3
\end{array}\right.
$$


where $\lambda=\lambda^{(3)}$. For any distinct elements $i, j, 3$ in $A$, in view of (5), (8), (9), (11), (12) and (14), we have

$$
\begin{aligned}
& \lambda P_{2}(f)\left(e_{i} \cdot e_{j}\right) \\
& =\lambda^{(3)} f\left(e_{i}\right) \cdot f\left(e_{j}\right) \\
& =\lambda_{i j}^{(3)} \alpha_{i 3} \alpha_{j 3} w_{i} \cdot w_{j} \\
& =\alpha_{i j} w_{i} \cdot w_{j} \\
& =T\left(e_{i} \cdot e_{j}\right) \\
& \lambda P_{2}(f)\left(e_{i} \cdot e_{3}\right) \\
& =\lambda f\left(e_{i}\right) \cdot f\left(e_{3}\right) \\
& =\lambda\left(\alpha_{i 3} w_{i}\right) \cdot\left(\frac{1}{\lambda} w_{3}\right) \\
& =\alpha_{i 3} w_{i} \cdot w_{3} \\
& =T\left(e_{i} \cdot e_{3}\right) \\
& \lambda P_{2}(f)\left(e_{i}^{2}-e_{3}^{2}\right) \\
& =\lambda^{(3)} f\left(e_{i}\right)^{2}-\lambda^{(3)} f\left(e_{3}\right)^{2} \\
& =\lambda_{i j}^{(3)} \alpha_{i 3}^{2} w_{i}^{2}-\lambda^{(3)}\left(\frac{1}{\lambda^{(3)}}\right)^{2} w_{3}^{2} \\
& =\lambda_{i j}^{(k)} \alpha_{i k}^{2} w_{i}^{2}-\frac{1}{\lambda^{(3)}} w_{3}^{2} \quad \text { where } k \notin\{i, j, 3\} \\
& =\lambda_{i j}^{(k)} \alpha_{i k}^{2} w_{i}^{2}-\frac{1}{\lambda_{i k}^{(3)}} w_{3}^{2} \\
& =\lambda_{i j}^{(k)} \alpha_{i k}^{2} w_{i}^{2}-\lambda_{i 3}^{(k)} \alpha_{3 k}^{2} w_{3}^{2} \\
& =\lambda^{(k)}\left(\alpha_{i k}^{2} w_{i}^{2}-\alpha_{3 k}^{2} w_{3}^{2}\right) \\
& =T\left(e_{1}^{2}-e_{3}^{2}\right) \\
& =
\end{aligned}
$$

Therefore $T=\left.\lambda P_{2}(f)\right|_{Z_{0}\left(U^{(2)}\right)}$ and $f$ is injective.

Case 2: $\left\langle w_{i}\right\rangle=\left\langle w_{j}\right\rangle$ for some distinct $i, j$.

Let $w=w_{1}$. Without loss of generality, we may assume that $w_{1}=w_{2}$. We first show that

$$
T\left(e_{i} \cdot\left\langle e_{i}\right\rangle^{\perp}\right) \subseteq w \cdot W
$$

for all $i \in A$.

If $w_{3}$ and $w$ are linearly independent, then

$$
T\left(e_{1} \cdot e_{3}\right)=\alpha w \cdot w_{3}
$$

for some $\alpha \in F \backslash\{0\}$. Similarly, $T\left(e_{2} \cdot e_{3}\right)=\beta w \cdot w_{3}$ for some $\beta \in F \backslash\{0\}$. Hence

$$
T\left(e_{3} \cdot\left(\beta e_{1}-\alpha e_{1}\right)\right)=0 \text {. }
$$

This contradicts the hypothesis that $\rho\left(T\left(e_{3} \cdot\left(\beta e_{1}-\alpha e_{1}\right)\right)\right)=2$. Thus $\left\langle w_{3}\right\rangle=\langle w\rangle$. Likewise, $\left\langle w_{k}\right\rangle=\langle w\rangle$, for all $k \in A$. Therefore, we can conclude that 


$$
T\left(e_{i} \cdot\left\langle e_{i}\right\rangle^{\perp}\right) \subseteq w \cdot W
$$

for all $i \in A$.

Now we show that

$$
T\left(e_{i}^{2}-e_{j}^{2}\right) \in w \cdot W
$$

for all distinct $i, j$.

Let

$$
\begin{aligned}
& T\left(e_{1} \cdot e_{3}\right)=w \cdot z_{1}, \\
& T\left(e_{2} \cdot e_{3}\right)=w \cdot z_{2}, \\
& T\left(e_{1} \cdot e_{4}\right)=w \cdot z_{3}, \\
& T\left(e_{2} \cdot e_{4}\right)=w \cdot z_{4},
\end{aligned}
$$

where $z_{1}, z_{2}, z_{3}, z_{4} \in W$. Let

$$
M=\left\langle\left(e_{1}+e_{2}\right) \cdot\left(e_{1}-e_{2}\right),\left(e_{1}+e_{2}\right) \cdot e_{3},\left(e_{1}+e_{2}\right) \cdot e_{4}\right\rangle .
$$

Then $\left\langle e_{1}-e_{2}, e_{3}, e_{4}\right\rangle$ is a three dimensional subspace of $\left\langle e_{1}+e_{2}\right\rangle^{\perp}$ and in view of Lemma 3.1, $\operatorname{dim} T(M)=3$ and $T(M) \subseteq v \cdot W$ for some $v \in W$.

Let

$$
\begin{aligned}
& T\left(\left(e_{1}+e_{2}\right) \cdot\left(e_{1}-e_{2}\right)\right)=v \cdot v_{1}, \\
& T\left(\left(e_{1}+e_{2}\right) \cdot e_{3}\right)=v \cdot v_{2}, \\
& T\left(\left(e_{1}+e_{2}\right) \cdot e_{4}\right)=v \cdot v_{3},
\end{aligned}
$$

where $\operatorname{dim}\left\langle v_{1}, v_{2}, v_{3}\right\rangle=3$. On the other hand,

$$
\begin{aligned}
T\left(\left(e_{1}+e_{2}\right) \cdot e_{3}\right) & =T\left(e_{1} \cdot e_{3}\right)+T\left(e_{2} \cdot e_{3}\right) \\
& =\left(z_{1}+z_{2}\right) \cdot w . \\
T\left(\left(e_{1}+e_{2}\right) \cdot e_{4}\right) & =T\left(e_{1} \cdot e_{4}\right)+T\left(e_{2} \cdot e_{4}\right) \\
& =\left(z_{3}+z_{4}\right) \cdot w .
\end{aligned}
$$

Since $\operatorname{dim} T(M)=3, z_{1}+z_{2}$ and $z_{3}+z_{4}$ are linearly independent. Then by comparing the two expressions for $T\left(\left(e_{1}+e_{2}\right) \cdot e_{3}\right)$ and $T\left(\left(e_{1}+e_{2}\right) \cdot e_{4}\right)$, clearly $\langle w\rangle=\langle v\rangle$ and we obtain

$$
T\left(e_{1}^{2}-e_{2}^{2}\right) \in w \cdot W \text {. }
$$

Likewise,

$$
T\left(e_{i}^{2}-e_{j}^{2}\right) \in w \cdot W
$$

for all distinct $i, j$. Now we have proved that

$$
T\left(e_{i} \cdot\left\langle e_{i}\right\rangle^{\perp}\right) \subseteq w \cdot W
$$

for all $i \in A$, and

$$
T\left(e_{i}^{2}-e_{j}^{2}\right) \in w \cdot W
$$

for all distinct $i, j$. Therefore $\operatorname{Im} T \subseteq w \cdot W$.

Remark. We conjecture that Theorem 3.2 is also true when $\operatorname{dim} U=3$. If $\operatorname{dim} U=2$, Theorem 3.2 is no longer true. 
Example Let $U$ be a 2-dimensional Euclidean space with an orthonormal basis $\left\{e_{1}, e_{2}\right\}$. Let $T: Z_{0}\left(U^{(2)}\right) \rightarrow U^{(2)}$ be a linear mapping such that $T\left(e_{1}^{2}-e_{2}^{2}\right)=e_{1} \cdot e_{2}$ and $T\left(e_{1} \cdot e_{2}\right)=e_{1}^{2}-e_{2}^{2}$. Then clearly $T$ is a rank 2 preserver. However $T$ is neither of the form (i) nor the form (ii) in Theorem 3.2.

Now we state Theorem 3.2 in matrix language when the dimension of $U$ and $W$ are finite.

Corollary 3.3 Let $n$ and $m$ be positive integers and let $L$ be a rank 2 preserver from $Z_{0}\left(S_{n}(F)\right)$ to $S_{m}(F)$. If $n \geq 4$, then one of the following holds:

(i) There exists a rank $n m \times n$ matrix $P$ such that $L(A)=\lambda P A P^{t}$ for all $A \in Z_{0}\left(S_{n}(F)\right)$ where $\lambda \in F \backslash\{0\}$;

(ii) There exists a nonsingular $m$-square matrix $Q$ such that

$$
\operatorname{Im} L \subseteq\left\{Q\left(\begin{array}{cccc}
c_{1} & c_{2} & \cdots & c_{m} \\
c_{2} & 0 & \cdots & 0 \\
\vdots & \vdots & \ddots & \vdots \\
c_{m} & 0 & \cdots & 0
\end{array}\right) Q^{t}: c_{j} \in F, j=1,2, \ldots, m\right\}
$$

The author would like to thank the referees for valuable comments.

\section{References}

1. P. Botta, S. Pierce, W. Watkins, Pacific J. Math. 104(1), 39 - 45 (1983)

2. C.K. Li S. Pierce, Canad. Math. Bull. 37(3), 374 - 383 (1994)

3. W. Watkins, J. Algebra 179, 549 - 596 (1996)

4. W. Watkins, Linear Algebra Appl. 250, 31 - 38 (1997)

5. M.H. Lim, Linear Algebra Appl. 430, 2982 - 2996 (2009) 